\section{SIMULATION OF LOCAL BLOOD FLOW IN HUMAN BRAIN UNDER ALTERED GRAVITY}

\author{
Chang Sung Kim (1), Cetin Kiris (2), and Dochan Kwak (2)
}

(1) Research Institute for Advanced Computer Science NASA Ames Research Center

Moffett Field, CA 94305-1000

(2) NASA Advanced Supercomputing Applications Branch NASA Ames Research Center

Moffett Field, CA 94305-1000

\section{INTRODUCTION}

Blood circulation as well as body fluids distribution undergoes significant adaptation during and after space flight. Much study on physiological changes under weightlessness has been performed since the early days of the space program. In particular, cardiovascular research in conjunction with the space shuttle program has included diverse physiological functions affected by the nervous system such as heart rate, blood pressure, hormone release, and respiration. The altered cardiac output due to adaptation during flight and deconditioning after the flight will impact blood circulation in the human body. Especially, this altered blood supply in the brain and consequent oxygen supply to certain parts of the brain will make nonnegligible impact on long-duration flight. To assess the impact of changing gravitational forces on human space flight, it will be essential to quantify the flow characteristics in the brain under varying gravity conditions. Analysis of blood circulation in brain as well as other parts of human body requires the capability to analyze flow in large arteries and capillaries.

In addition to the altered gravitational forces, specific shapes and connections of arteries in the brain vary in the human population (Cebral et al., 2000; Ferrandez et al., 2002). Considcring the geometric variations, pulsatile unsteadiness, and moving walls, computational approach in analyzing altered blood circulation will offer an economical alternative to experiments. This paper presents a computational approach for modeling the local blood flow through the human brain under altered gravity. This computational approach has been verified through steady and unsteady experimental measurements and then applied to the unsteady blood flows through a carotid bifurcation model and an idealized Circle of Willis (CoW) configuration under altered gravity conditions.

\section{NUMERICAL APPROACH}

The flow through the heart and blood vessels is unsteady, viscous, and incompressible. Blood flows through the heart and larger vessels have been assumed Newtonian. For instance, the Newtonianassumed blood flows through artificial heart devices were simlated using the INS3D code (Kiris et al., 1997). In the present study, the flows of interest are assumed non-Newtonian to account for the shear thinning behavior of the human blood. Governing equations are the three-dimensional, unsteady, incompressible Navier-Stokes equations coupled with non-Newtonian models. The solution algorithm is based on the method of artificial compressibility and dual time stepping. Several types of non-Newtonian models were implemented into the INS3D code and assessed through steady and unsteady computations. Among them, a Carreu-Yasuda model (Gijsen et al., 1999) is adopted for the computation of non-Newtonian flows in the present study. This model describes the shear thinning behavior of blood flows with asymptotic apparent viscosities at zero and infinite shear rates.

For moving wall boundaries, a first-order approximate model for arterial wall distensibility is adopted based on the assumption of a thin-walled and linearly elastic channel. Compared with finite element methods (Perktold and Rappitsch, 1995; Zhao et al., 2002), this approach was found to be a standard first-order approximation to the complex behavior of the arterial wall (Steinman and Ethier, 1994). Embedding in and tethering to surrounding structures is thought to reduce the arterial longitudinal motion to a minimum. In the present study, therefore, blood vessels are assumed to have circular, thin and elastic walls with negligible Iongitudinal motion. To make the problem computationally manageable, minor arteries such as arterioles, venules and capillaries need to be truncated. A novel algorithm for outflow boundary conditions is imposed at the truncated positions by utilizing the analogy of arterial branches to electric circuits and Poiseuille's theorem. For the problem with multiple outflow boundaries, a hybrid approach is adopted by combining this algorithm with the pressure waveform measurement at a reference point.

An MPI-OpenMP hybrid version of INS3D code has been extended with implementation of those numerical approaches, and computational experiments have been conducted with the parallelized SGI workstation cluster.

\section{RESULTS AND DISCUSSION \\ Effect of Wall Distensibility under Altered Gravity}

The blood flow through a carotid bifurcation model is computed with the mean flow rate of $8 \mathrm{ml} / \mathrm{s}$ in the common carotid artery (CCA). The Reynolds number based on CCA diameter is 388 . The effect of wall distensibility under different gravity conditions is demonstrated in Fig. 1.

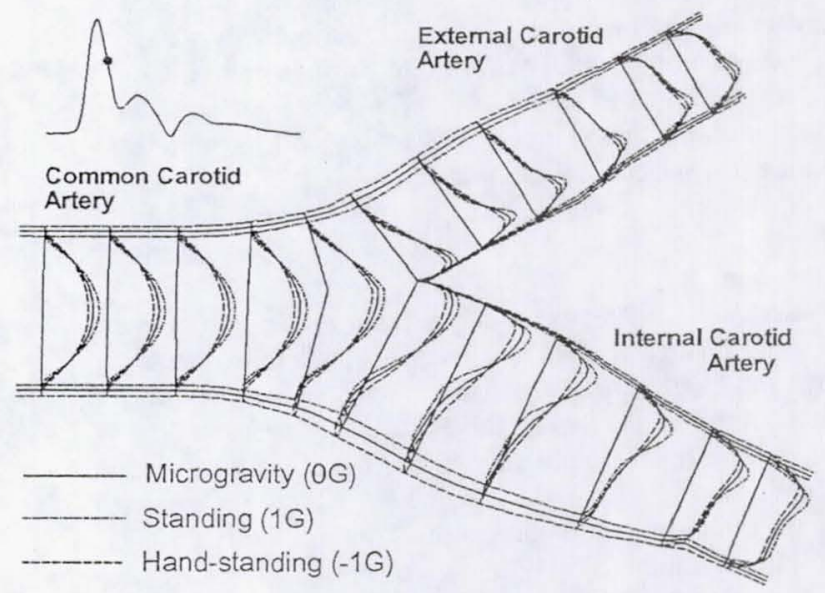

Fig. 1 Gravitational effects on wall motion and axial velocity profiles at systolic deceleration phase.

The computed result under microgravity (or approximately in supine posture under normal gravity) is compared with those of both standing and hand-standing cases simultaneously. In standing posture, the normal gravity force or $1 G$ applies downward. In hand-standing posture, the gravity applies toward the head, which is nominally $-1 G$. The relative diameter change of $-1 G$ case to $1 G$ case is up to 14 percent at the systolic deceleration phase, which approximately corresponds to 41 percent reduced vessel resistance from the Poiseuille's theorem. Wall shear stress distributions in Fig. 2 indicate 
that the reversed flow region along the sinus of the internal carotid artery (ICA) becomes narrower as the diameter increases. It is observed that the altered gravity has a significant influence on the wall motion and flow patterns throughout the pulse cycle.
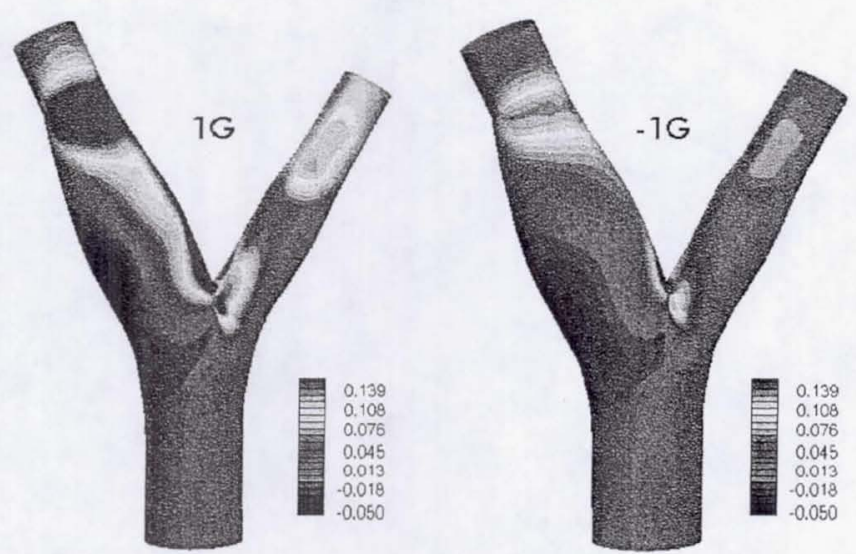

Fig. 2 Gravitational effect on wall shear stress distribution at systolic deceleration phase.

\section{Blood Circulation in Human Brain}

To provide the fundamental understanding of gravitational effects on cerebral hemodynamics and the mechanism of collateral circulation in the brain, an idealized CoW configuration was designed based on the statistical anatomy with minor arteries truncated. The mean flow rate through the CoW model is $3.5 \mathrm{ml} / \mathrm{s}$ in the vertebrobasilar artery (VBA). The Reynolds number is 240 based on the VBA diameter and the mean inflow velocity. To demonstrate the effects of altered gravity, the computed result under the normal gravity of $1 \mathrm{G}$ was simulated and compared with those under microgravity or zero gravity. For both cases, the total inflow rate through left and right ICAs, and VBA is assumed to be the same because the autoregulation is known to control the blood flow constant regardless of altered pressure to a certain extent. In comparison with the microgravity result, the normal gravity case shows a maximal contraction of the vessel diameter up to about 12 percent due to the decreased blood pressure in the brain.

Even though one of main arteries in the brain is stenosed or even missing, the distal smaller arteries can receive blood from the other arteries through the CoW. To simulate this interesting mechanism of "collateral circulation" in the brain, the left ICA is presumed 66.7 percent stenosed. It means that only one third of the blood through the right ICA is delivered to the CoW through the left ICA as indicated in Fig. 3. Unlike the balanced configuration case, the mass flux through the posterior communicating artery $(\mathrm{PCoA})$ is increased to compensate about 22 percent for the deficiency. Also the blood flow through anterior communicating artery $(\mathrm{ACOA})$ is not zero. Instead, the mass flux through the proximal part of the left anterior cerebral artery (ACA) is very low in order to distribute blood to the left middle cerebral artery (MCA) and the left ACA simultaneously. This result demonstrates the mechanism of collateral circulation in the brain.

\section{CONCLUSION}

It was noticed that the effect of wall deformability on internal flows is remarkably augmented due to the gravity. For the realistic carotid artery model, the wall distensibility has a quantitative influence on the unsteady flow pattern and wall shear stress distribution.

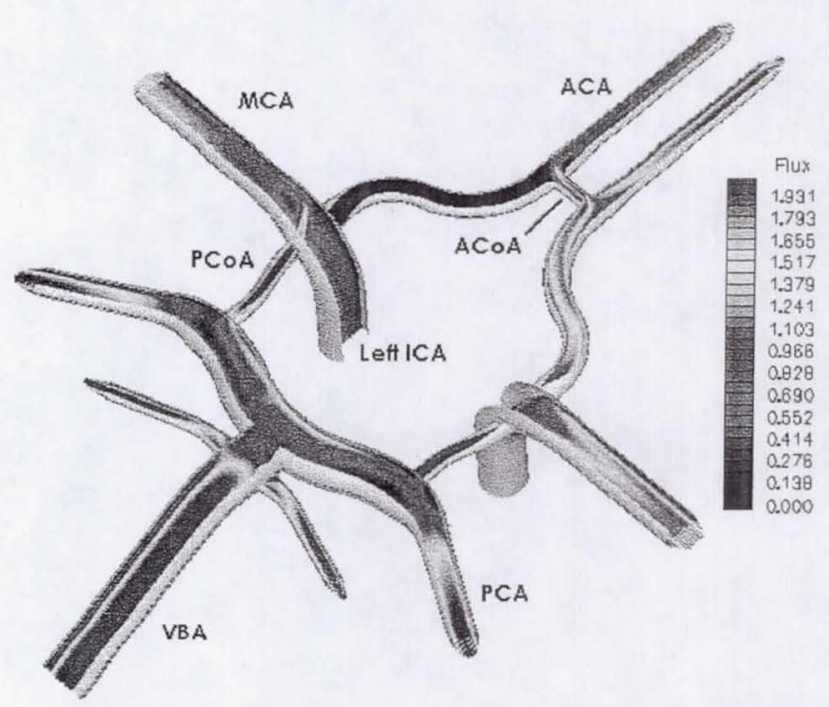

Fig. 3 Collateral circulation through the CoW with left ICA $66.7 \%$ stenosed.

In addition, not only the effect of altered gravity on cerebral circulation but also the mechanism of collateral circulation thorough the CoW were demonstrated. The solution procedure based on parallel computation adopted in this study has shown its capability that can model the multi-scale hemodynamics for the various types of the human circulatory system.

\section{REFERENCES}

Cebral, J. R., Lohner, R., and Burgess, J., 2000, "Computer Simulation of Cerebral Artery Clipping: Relevance to Aneurysm Neuro-Surgery Planning," Proc. ECCOMAS, Sep. 11-14, Barcelona, Spain.

Ferrandez, A., David, T., and Brown, M. D., 2002, "Numerical models of Auto-regulation and Blood Flow in the Cerebral Circulation," Computer Methods in Biomechanics and Biomedical Engineering, 5 (1), pp. 7-20.

Gijsen, F. J. H., van de Vosse, F. N., and Janssen, J. D., 1999a, "The Influence of Non-Newtonian Properties of Blood on the Flow in Large Arteries: Steady Flow in a Carotid Bifurcation Model," Journal of Biomechanics, 32, pp.601-608.

Kiris, C., Kwak, D., Rogers, S., and Chang, I.-D., 1997, "Computational Approach for Probing the Flow Through Artificial Heart Devices," ASME Journal of Biomechanical Engineering, 119, pp. 452-460.

Perktold, K. and Rappitsch, G., 1995, "Computer Simulation of Local Flow and Vessel Mechanics in a Compliant Carotid Artery Bifurcation Model," Journal of Biomechanics, 28 (7), pp. 845-856.

Steinman, D. A. and Ethier, C. R., 1994, "Effect of Wall Distensibility on Flow in a Two-Dimensional End-to-Side Anastomosis," ASME Journal of Biomechanical Engineering, 116, pp. 294-301.

Zhao, S. Z., Ariff, B., Long, Q., Hughes, A. D., Thom, S. A., Stanton, A. V., and Xu, X. Y., 2002, "Inter-individual Variations in Wall Shear Stress and Mechanical Stress Distributions at the Carotid Artery Bifurcation of Healthy Humans," Journal of Biomechanics, 35 , pp. 1367-1377. 